\title{
„Sprachwissenschaft [...] etwas für Leute, welche die Pedanterie zur Mathematik hätten, nicht jedoch die Intelligenz. Leute, die sich ihre eigene notdürftige Logik erfänden" -
}

\author{
Auf den Spuren des Ausdrucks von Emotionen
}

\author{
Monika WOLF
}

\begin{abstract}
"Sprachwissenschaft [...] etwas für Leute, welche die Pedanterie zur Mathematik hätten, nicht jedoch die Intelligenz. Leute, die sich ihre eigene notdürftige Logik erfänden"-

Tracking down the expression of emotion

In order to track down the devices used in the verbal expression of emotions, it is helpful to proceed semasiologically, for example by searching for emotive expressions in authentic texts. In texts written in everyday language, emotions are frequently verbalized without designating any particular emotion. Therefore, the pertinent linguistic devices should be identified with the aid of a small corpus, because only in this way can corpus-linguistic principles of exhaustivity and context-sensitivity be fulfilled. This brings into focus syntactic devices, which have so far (for the most part) been neglected by research in the field of emotiology.
\end{abstract}

Keywords: emotions, modality, small corpus theory, syntax

DOI: $10.15452 /$ StudiaGermanistica.2021.29.0005

\section{Einleitung: Authentische Sprachdaten}

In dem Roman Die Vermessung der Welt von Daniel Kehlmann diskutieren die Protagonisten Alexander von Humboldt und sein Reisegefährte Aimé Bonpland am Orinoko über den Konquistador Aguirre. Bonpland äußert, dass Aguirre der erste Erforscher des Flussgebietes sei, worauf Humboldt erwidert:

Dieser traurige Mann habe gar nichts erforscht, sagte Humboldt.

(Die Vermessung der Welt: 138) 
Somit gibt er seine Einschätzung zur Bedeutung Aguirres als Forscher kund. Er verwendet dabei ein Adjektiv, das allgemein zum Emotionswortschatz gezählt wird. Dennoch beschreibt er damit nicht die emotionale Befindlichkeit einer Person, sondern offenbart seine Einstellung als Sprecher zu ihr. Es liegt dennoch ein hoher Grad an Expressivität vor, d.h. der Sprecher drückt etwas über seine „Innerlichkeit“ aus, wie Karl Bühler es formuliert. Ihm zufolge ist der Ausdruck neben dem Appell und der Darstellung eine der drei Grundfunktionen des sprachlichen Zeichens, die er in seinem Organon-Modell beschreibt (1965:28): Das Sprachliche Zeichen ist „Symbol kraft seiner Zuordnung zu Gegenständen und Sachverhalten, Symptom (Anzeichen, Indicium) kraft seiner Abhängigkeit vom Sender, dessen Innerlichkeit es ausdrückt, und Signal kraft seines Appells an den Hörer, dessen äußeres oder inneres Verhalten es steuert wie andere Verkehrszeichen.“ Zur Symbolfunktion könnte man auch ,referentielle“ Funktion sagen (vgl. Jakobson 2007:165). Es geht um das Objekt, den Referenten der Rede. Die Signalfunktion bezeichnet Jakobson als „konative Sprachfunktion“: „Die konative Funktion findet ihren reinsten grammatischen Ausdruck im Vokativ und im Imperativ (ibid.). Die „Innerlichkeit“, ausgedrückt durch das Symptom, betrifft Meinungen, Wünsche und Gefühle/Emotionen. Mit der Ausdrucksfunktion ist also die Möglichkeit und Fähigkeit benannt, die emotive Modalität zu realisieren.

Die Modalität, also die subjektive Stellungnahme eines Sprechers zu den verbalisierten Sachverhalten kann epistemisch (Verstand, Wissen, Kognition), volitiv (Wille, Wunsch) und emotiv (Gefühl, Gemüt) ausfallen. Bereits in der antiken Philosophie und Psychologie (bes. Platon) wird der Charakter eines Menschen als Zusammenwirken von Verstand, Wille und Gemüt beschrieben, das unsere Einstellungen zu Sachverhalten wesentlich bestimmt. Brunot übernimmt in La pensée et la langue (1922:507) diese Einteilung, indem er notre jugement, notre sentiment und notre volonté als „modalités de l'idée“ bezeichnet. Diese drei Kategorien der Modalität werden wiederum von Charles Bally ( ${ }^{4}$ 1965:35f.) aufgegriffen. Schließlich findet sich diese Auffassung von Modalität auch im Organon-Modell Karl Bühlers: Die Darstellungsfunktion entspricht der epistemischen Modalität, die Appellfunktion der volitiven Modalität und Ausdrucksfunktion der emotionalen Modalität.

In jeder Äußerung sind verschiedene Modalitäten realisiert - mit unterschiedlichen Schwerpunkten, d.h. jeder Sprecher äußert immer auch eine subjektive Stellungnahme zum verbalisierten Sachverhalt. Somit kann man sagen, dass die emotionale Modalität in fast allen Texten in irgendeiner Form vorkommt. Daneben kann man das ,Emotionalisieren' mit dem Bühler'schen Organonmodell modellieren: Emotionen werden ausgedrückt (Ausdruck), über sie wird gesprochen (Darstellung) und sie werden durch sprachliche Mittel beim Rezipienten erzeugt (Appell). Da ich Emotionalität als Teil der Modalität verstehe, geht es in diesem Artikel vorrangig um den (sprachlichen) Ausdruck von Emotionalität, also um die expressive Funktion des sprachlichen Zeichens.

Um die Mittel, mit denen Emotionalität versprachlicht wird, zu erfassen, ist es sinnvoll, korpuslinguistisch und semasiologisch vorzugehen, d.h. in authentischen Texten die emotiven Ausdrücke zu suchen. In solchen authentischen Texten (nicht nur in alltagssprachlichen Texttypen, sondern auch in sogenannter , hoher Literatur") wird häufig Emotionalität ausgedrückt, ohne dass der genaue emotionale Gehalt einer Äußerung bestimmt werden kann. Dabei spielt der Kontext in der Regel eine entscheidende Rolle. Es bietet sich daher an, die einschlägigen sprachlichen Mittel mithilfe eines kleinen Korpus zu ermitteln und dabei semasiologisch vorzugehen, da nur so den korpuslinguistischen Prinzipien der Exhaustivität und der Kontextsensitivität entsprochen werden kann. Andererseits wird ein weiteres methodisches Postulat korpuslinguistischen Vorgehens, die Frequenzorientiertheit, hier keine Rolle spielen. Denn es geht nicht um einzelne Wörter oder Wendungen und deren Frequenz in den Korpustexten, sondern um satz- und textgrammatische Phänomene sowie um eine Reihe von Partikeln, die bei der Schaffung der, emotionalen Stimmung' zusammenwirken. ,Korpuslinguistisch' heißt hier also, dass sprachliche Verfahrensweisen in authentischen Texten in ihren Kontexten analysiert und beschrieben werden. Auf diese Weise rücken auch vor allem auf syntaktischer Ebene Mittel in den Fokus, die bisher in der emotionslinguistischen Forschung kaum berücksichtigt wurden.

Laut Wilce (2009:3) sind Emotionen potenziell in allen Ebenen der Sprache zu finden: „All speaking and writing is inherently emotional to a greater or lesser extent; objective, distant coolness 
is an emotional stance“. Es empfiehlt sich, literarische Texte als Grundlage für solche korpuslinguistischen Untersuchungen zu nehmen. In solchen Texten stellen die Emotionen ein ,wesentliches Charakteristikum des jeweiligen Textweltmodells" dar (vgl. Schwarz-Friesel 22013:218; dieses Modell konzeptualisiert jeden literarischen Text als eigene Welt). ${ }^{1}$ In diesem Sinn kann auch Coserius Meinung ( $\left.{ }^{3} 1994: 146-149\right)$, dass nur die Literatur als Kunstwerk alle Möglichkeiten der Sprache in ihrer gesamten Funktionalität entfaltet, als korpuslinguistisches Postulat verstanden werden.

Das Korpus für die folgenden Analysen besteht aus drei Passagen aus Kehlmanns Roman. Lineare Textanalysen werden alle lexikalischen, morphologischen und syntaktischen Emotionalitätsausdrücke ermitteln. Dadurch wird ein sehr breites Spektrum von Emotionsausdrücken sichtbar werden, weil durch die lineare Textanalyse nicht nur das Bekannte gefunden wird. Die einzelnen Möglichkeiten, Emotionen zu versprachlichen, werden anschließend kategorisiert und in Hinblick auf ihre kommunikativen Funktionen untersucht. Es geht also nicht um emotionstheoretische Details oder Neuerungen, sondern um sprachliche Daten.

\section{Lineare Textanalysen}

In dem zu Beginn dieses Beitrags zitierten Beispiel äußert sich der Sprecher nicht explizit zu seiner emotionalen Befindlichkeit, aber dennoch manifestiert er seine gefühlsmäßige Einstellung zum Konquistador. Dies geschieht nicht mit der bloßen Verwendung eines Emotionswortes, sondern wird aus dem Kontext erkenntlich. Im Folgenden werden anhand von ausgewählten Passagen des Romans Die Vermessung der Welt verschiedene Möglichkeiten des Emotionsausdrucks untersucht und kategorisiert.
Der Fluß, sagte Julio, dulde keine Menschen. Bevor Aguirre sich hierhin aufgemacht habe, sei er bei Verstand gewesen. Erst hier sei es ihm eingefallen, sich zum Imperator zu erklären.
Ein verrückter Mörder, sagte Bonpland, der erste Erforscher des Orinoko! Das ergebe Sinn.
Dieser traurige Mann habe gar nichts erforscht, sagte Humboldt. Ebensowenig erforsche ein Vogel die Luft oder ein Fisch das Wasser.
Oder ein Deutscher den Humor, sagte Bonpland.
Humboldt sah ihn mit gerunzelten Brauen an.
Nur ein Witz, sagte Bonpland.
Aber ein ungerechter. Ein Preuße könne sehr wohl lachen. In Preußen werde viel gelacht. Man solle nur an die Romane von Wieland denken oder die vortrefflichen Komödien von Gryphius. Auch Her- der wisse einen guten Scherz wohl zu setzen.
Daran zweifle er nicht, sagte Bonpland müde.
Dann sei es ja gut, sagte Humboldt und kraulte das von den Insektenstichen blutige Fell des Hundes.
(Die Vermessung der Welt: 137 ff.)

Diese Szene gibt eine Unterhaltung dreier Männer verschiedener Nationalitäten wieder, die gemeinsam den Orinoko entlang reisen, um ein bisher unbekanntes Gebiet zu erforschen. Die drei Personen werden von Moskitos geplagt. Julio versucht, dieses quälende Phänomen zu erklären, er tut dies mit einem Deklarativ-/Darstellungssatz: Der Fluß, sagte Julio, dulde keine Menschen. Der Fluss wird geradezu vermenschlicht, er ist ein Wesen, das einen Willen hat, der andere Wesen zulässt oder abweist. Diese Äußerung wird als indirekte Rede wiedergegeben, d.h. der Erzähler distanziert sich von dieser Äußerung, indem er keine Gewähr für deren Wahrheit übernimmt. Die Rede wird so Teil der Erzählung, was sich vor allem in den Personalpronomina der 3. Person widerspiegelt. Es ist ein Kennzeichen des ganzen Romans, dass er keine direkte Redewiedergabe enthält; allenfalls verblose Äußerungen könnte man so lesen.

Auffällig ist, dass jede Figur in erster Linie von ihren eigenen Erfahrungen und Erkenntnissen ausgeht, auch wenn sie miteinander zu sprechen scheinen. Dies ändert sich in dem Augenblick, in

Vgl. Oatley (2004:98): „Fiction contributes to the building of characters by means of emotions.“ Vgl. auch Bestgen (1994), Konstantinidou (1997:105f). und Herding/Stumpfhaus (2004). 
dem sie gewissermaßen auf einen gemeinsamen Gegenstand stoßen. Das ist hier der Konquistador Lope de Aguirre, der in dem Gespräch als bekannt vorausgesetzt wird, weil nur sein Name ohne jeden kommentierenden Zusatz genannt wird. Julio verwendet das Verb einfallen bei der Erwähnung von Aguirres Revolte und Errichtung eines Terrorregimes und drückt damit aus, dass er dessen Aktionen für eine plötzliche, fast launenhafte Anwandlung eines Geistesgestörten hält. So gibt er eine negative Beurteilung, wenn nicht gar Verachtung kund.

Der Botaniker Aimé Bonpland charakterisiert Aguirre mit einer verblosen Setzung, in der das Verbum dicendi als Redeeinleitung steht, am Ende kommt ein Ausrufezeichen als Signal dafür, dass es sich um eine expressive Äußerung handelt. Verblose Setzungen sind im weiteren Sinn Aposiopesen, Formen der syntaktischen Reduktion, also (modal, emotional, ...) markierte, aber , ungesättigte‘ Satzformen, die der ,Sättigung ' durch den Kontext und die Konsituation bedürfen. Bonpland wertet mit der ersten Setzung den Konquistador deutlich negativ und legt - das Ausrufezeichen ist ein graphischer Ersatz für die Intonation - negative Emotionen in diese Äußerung. Würde der Satz lauten Ich halte Aguirre für einen verrückten Mörder, dann wäre die subjektive Geltung dieser Wertung zwar deutlich ausgedrückt, aber nicht so expressiv formuliert. Nach dem Verbum dicendi kommt eine eher anerkennende Wertung, die in der Folge von Humboldt relativiert, wenn nicht negiert wird. Wir haben hier somit eine häufig anzutreffende Art der Emotionalisierung vor uns, die eben nur (allgemeine) Emotionalität ausdrückt, aber nicht deren Inhalt, den wir meistens aus dem Wortlaut der emotionalen Äußerung erschließen müssen. Die Grammatik kann nicht für jede einzelne Emotion ein eigenes Mittel zur Verfügung stellen, dazu ist der Wortschatz viel besser gerüstet. Durch die Verblosigkeit der Setzung wird auch die Opposition zwischen direkter und indirekter Rede neutralisiert; die Äußerung wirkt viel unmittelbarer und emotionaler.

Im Anschluss daran äußert sich Humboldt in einem Deklarativsatz, charakterisiert aber Aguirre durch das Syntagma dieser traurige Mann. Damit ist nicht gemeint, dass Aguirre ein Melancholiker war; das Adjektiv traurig hat hier in Anlehnung an die Formen eine traurige Figur die Bedeutung „kümmerlich, erbärmlich“. ${ }^{2}$ Der Konquistador wirkt deshalb so erbärmlich, weil er nach Meinung Humboldts keinerlei Forschungsleistungen erbracht hat. Die Gradpartikel gar, die das Negationspronomen nichts steigert (vgl. Métrich/Faucher 2009:378.), signalisiert trotz der unmarkierten Satzform einen hohen Grad an Expressivität in der Äußerung Humboldts. Die beiden Vergleiche aus dem Bereich der Tierwelt bringen seine negative Beurteilung und somit seine emotionale Einstellung wiederum verstärkend zum Ausdruck.

Man kann jetzt schon beobachten, dass Humboldt und Bonpland auf unterschiedliche Weise unterschiedliche Emotionen ausdrücken: Humboldt spricht, syntaktisch gewissermaßen neutral, Urteile in Deklarativsätzen. Bonpland hingegen wertet permanent in verschiedenen Formen syntaktischer Reduktion. So setzt er scheinbar eine Äußerung Humboldts fort: Oder ein Deutscher den Humor. Diese verblose Äußerung Bonplands ist nicht eine expressive Setzung, sondern eine Ersparung von Satzgliedern aufgrund von Vorerwähntheit. Dieses sprachökonomische Mittel erlaubt es, nur die Wörter zu äußern, auf die es dem Sprecher ankommt. Der kommunikativ wichtige Kontrast zur vorausgehenden Äußerung wird so deutlich. Der Erzähler lässt Humboldt an dieser Stelle nicht argumentativ oder zumindest sprachlich reagieren, sondern physiognomisch: Humboldt runzelt die Augenbrauen. Schon Charles Darwin bemerkt: „Menschen aller Rassen runzeln die Stirn, wenn sie in ihren Gedanken auf irgend welche Weise verworren werden“"(Darwin 1877:202.). Der Verhaltensforscher Desmond Morris nennt gerunzelte Augenbrauen als Zeichen für große Angst, erwähnt aber auch dass unter anderem das Gefühl von Wut diesem Gesichtsausdruck zugrunde liegt (Vgl. Morris 1994:52). Laut der Psychologen Ekman und Friesen liegt der Unterschied darin, ob die zusammengezogenen Augenbrauen gleichzeitig gesenkt oder gehoben sind (Ekman, Friesen 2003:68 ff., 82 f.). Jedoch sind eine präzisere Beschreibung der Bewegungen der einzelnen Gesichtsmuskeln und die entsprechenden naturwissenschaftlichen Kenntnisse für das Verständnis nicht nötig. Aufgrund des Kontexts, vor allem durch Humboldts folgende Äußerungen, dass er auch diese

https://www.duden.de/rechtschreibung/traurig [17.01.2021]. 
Äußerung von Bonpland zumindest missbilligt und es sich somit um einen gewissen Grad von Verärgerung handelt.

Bonpland nimmt Humboldts negative Gefühlsregung auch wahr, weswegen er versucht, mit einer Setzung die Gefühlslage zu entschärfen, in der die Fokuspartikel nur zur (De-) Emotionalisierung dient: Es handle sich um nichts Weiteres als eine belanglose Bemerkung, die zur allgemeinen Erheiterung führen sollte und daher kein Grund zu Verärgerung sein könne. Darauf setzt Humboldt das Dialogmittel der Ersparung von Vorerwähntem ein, um sowohl die adversative Konjunktion aber als auch das wertende Adjektiv ungerecht hervorzuheben. Anschließend argumentiert er wieder, indem er in Deklarativsätzen die Wertung Bonplands zu widerlegen versucht.

Bonpland hat offensichtlich kein großes Interesse an einer Fortführung des Dialogs und lenkt ein. Seine Zustimmung äußert er dieses Mal mit einem Deklarativsatz, was erkennen lässt, dass der Grad der emotionalen Erregung geringer ist. Allerdings charakterisiert der Erzähler diesen Akt der Äußerung mit dem Adjektiv müde, womit kein physischer Zustand beschrieben wird, sondern eine gefühlsmäßige Verfassung im Sinne von ,überdrüssig'. ${ }^{3}$ Dieses Beispiel verdeutlicht aufs Neue, wie Emotionalität in der Sprache nur durch den Kontext zum Vorschein kommt.

Das Gespräch dieser drei Männer findet in der Wildnis einer unerforschten Region fernab von jeglicher Zivilisation statt. Ganz anders verhält es sich mit dem folgenden Dialog zwischen Humbolds Bruder Wilhelm, dem großen Sprachwissenschaftler und bedeutenden Bildungspolitiker, und dem Mathematiker, Statistiker, Astronomen, Geodäten und Physiker Carl Friedrich Gauß im Weimarer Hoftheater nach einer Voltaire-Aufführung, also in einer förmlichen Situation. Die Figuren machen sich in diesem Textausschnitt miteinander bekannt, stehen also in einem ganz anderen Verhältnis zueinander als die drei Reisegefährten. Wie in jedem Dialog kommen auch hier Emotionen zum Ausdruck, von denen sich der Erzähler, wie sehr häufig in diesem Buch, durch die Form der indirekten Rede distanziert. Für Emotionen sind nur die Figuren zuständig:

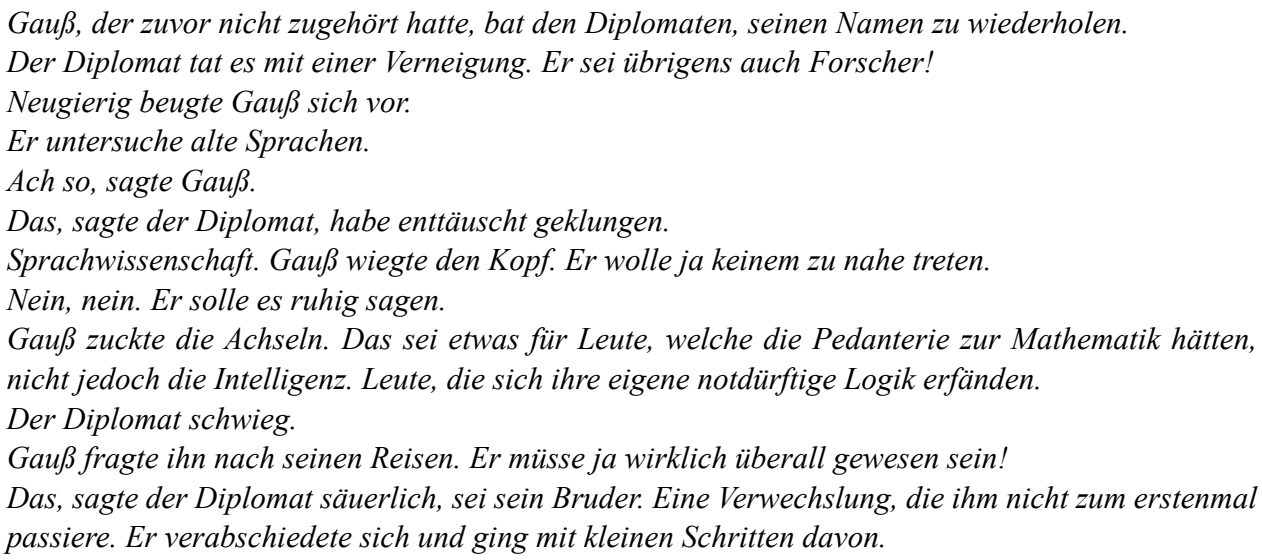
(Die Vermessung der Welt: 201)

Humboldt hat sich dem Mathematiker zuvor ausführlich vorgestellt, der sich aber kaum interessiert zeigt, was der Erzähler durch den attributiven Relativsatz der zuvor nicht zugehört hatte beschreibt. Ein Attribut, somit auch ein attributiver Nebensatz, charakterisiert das attribuierte Substantiv: Es ist also eine Charaktereigenschaft von Gauß, dass er nicht (immer) zuhört, dass er also keine Empathie zeigt und sich, wie der folgende Text zeigt, von Mitmenschen eher, genervt' fühlt. Diese Art von Emotionalität manifestiert sich nicht durch syntaktische Konstruktionen, sondern durch erzählerische Mittel, weswegen wir hier von ,narrativer Emotionalität' sprechen können.

Ganz anders verhält es sich mit Humboldts Befindlichkeit, der auf Gauß zugegangen war. Er ist nicht brüskiert, sondern wiederholt seinen Namen mit einer Verbeugung und bezeugt so seine

https://www.dwds.de/wb/m\%C3\%BCde [17.01.2021]. 
Hochachtung - er möchte den Dialog aufrechterhalten und seinen Gesprächspartner durch den Respekterweis positiv stimmen. Um dessen Interesse zu erwecken, verkündet er sogleich, dass er sozusagen ein Kollege sei. Das Ausrufezeichen dient als Hinweis auf Intonation und Stimmmodulation des Sprechers und somit auf dessen positive Erregtheit, die er auch bei Gauß erzeugen möchte. Zunächst ist diese Strategie auch erfolgreich, denn Gauß ändert seine Körperhaltung und sein plötzliches Interesse wird vom Erzähler mit dem Adjektiv neugierig beschrieben. Allerdings verhallt dieses wieder abrupt, als er den Gegenstand von Humboldts Forschung, von dem er offensichtlich nicht viel hält, erfährt.

Gauß reagiert wortkarg mit der Interjektion ach so. Das verbum dicendi der Erzählerinstanz deutet eine eher monotone Intonation des Sprechers an. Ach so ist hier sowohl Ausdruck des Verstehens als auch der Verwunderung. ${ }^{4}$ Der emotionale Gehalt von ach schwingt auch im zweiwortigen Ausdruck mit. In diesem Textausschnitt lässt der Kontext erkennen, dass es sich um Enttäuschung handelt, was Humboldt auch verbalisiert, wobei er mit dem Verb klingen auf die entsprechende Stimm-Modulation verweist.

Mit seiner Reaktion verrät Gauß seine Einstellung gegenüber Humboldts Fachdisziplin und sein Bemühen, nicht unhöflich zu erscheinen und seinen Gesprächspartner dadurch zu verärgern, was für ihn eher untypisch ist. Zunächst nennt er das Fach beim Namen, und seine zögernde Antwort zeigt, dass er nach den richtigen Worten sucht. Die Aussage Er wolle ja keinem zu nahe treten ist ein Versuch, höflich zu scheinen, d.h. dem Gegenüber sein ,Gesicht' zu lassen, und dient der prophylaktischen (De-)Emotionalisierung: Er versucht, das Hervorrufen von negativen Emotionen bei seinem Gesprächspartner zu vermeiden. Dabei bedient er sich der Metapher des Eindringens in die Intimsphäre des Gegenübers.

Humboldt wiederholt die Responsivpartikel nein, um eine beschwichtigende Wirkung zu erzielen und zu versichern, dass er nicht beleidigt sein werde. Das Gleiche drückt die Modalpartikel ruhig aus: Sie ,bringt in Sätzen, die eine Erlaubnis oder eine Aufforderung ausdrücken, die (u.U. nur vorgetäuschte) wohlwollende Haltung des Sprechers gegenüber dem Adressaten zum Ausdruck. Der Sprecher gibt damit kund, dass er gegen das betreffende Verhalten nichts einzuwenden hat.“ (Métrich/Faucher 2009:725). Er lädt also Gauß dazu ein, aufrichtig zu antworten.

Dieser zögert weiterhin und manifestiert seine negative Einstellung erst körpersprachlich. Achselzucken ist laut Duden eine „Gebärde des Nichtwissens oder der Gleichgültigkeit". 5 Schließlich beschreibt er Humboldts Disziplin mit den negativ konnotieren Wörtern Pedanterie und notdürftig und gibt somit kund, dass seiner Meinung nach die Sprachwissenschaft im Vergleich zu seinem eigenen Forschungsgegenstand kein ernstzunehmendes Fach sei.

Humboldt zeigt, dass er nun doch von dieser Einschätzung betroffen ist, indem er schweigt. Auch das Verstummen kann ein Emotionsausdruck eines Sprechers sein: "Schweigen ist in sozialen Situationen nicht bloß Leere; es vermittelt Bedeutungen.” (Kamper, Wulf 1992:7).

Um der peinlichen Situation zu entgehen, wechselt Gauß das Thema und wendet sich einer Sache zu, die ihn offensichtlich viel mehr interessiert als die Linguistik, denn er äußert sich in einem Deklarativsatz, der mit einem Ausrufezeichen markiert ist und verwendet die Modalpartikel wirklich um zu verdeutlichen, dass seine Aussage eine überraschende Angelegenheit betrifft (Vgl. Métrich, Faucher 2009:953 ff.). Damit drückt er aber nicht Überraschung, sondern Anerkennung, also seine positive Einstellung zum Sachverhalt aus.

Allerdings ist Humboldt, der sich zuvor von Gauß`abschätziger Zurückhaltung weniger beeindrucken ließ, nun doch endgültig brüskiert und beendet das Gespräch, nachdem er mit zwei Deklarativsätzen, die keinen Aufschluss auf seine Gefühlslage zulassen, Gauß` Irrtum aufklärt. Von seiner negativen Emotion erfährt der Leser durch das Adjektiv säuerlich in der Erzählerrede, das in seiner Bedeutung von ,missvergnügt ${ }^{‘}{ }^{6}$ zum Emotionswortschatz zählt. Der Kontext lässt hier

https://www.duden.de/rechtschreibung/ach [16.12.2020].

https://www.duden.de/rechtschreibung/Achselzucken [16.12.2020].

https://www.duden.de/rechtschreibung/saeuerlich [17.01.2021]. 
erkennen, dass Humboldt aufgrund der Verwechslung mit seinem Bruder aus Eifersucht oder wegen eines Minderwertigkeitskomplexes frustriert ist

Im Vergleich zum ersten Text ist der Erzähler viel stärker involviert. Er lässt die Figuren nicht nur für sich sprechen, indem er detaillierter den Körperausdruck und ihre Äußerungen mit Adverbialen aus dem Bereich des Emotionswortschatzes beschreibt, womit der Leser einen Hinweis auf die Stimm-Modulation des Sprechers erhält. Die Emotionalität äußert sich dadurch unmittelbarer. Gleichzeitig zeichnet sich die Figurensprache durch eine schwächere Expressivität aus. Gefühle, vor allem negative, sollen in diesem Kontext nicht offen zur Schau getragen werden.

Wie sehr sich Gauß in der höfischen Gesellschaft für seine Verhältnisse zurückhält, zeigt ein anderer Text aus dem Beginn des Romans, der sich im familiären Umfeld abspielt, wo er eine ganz andere Rolle hat. Es handelt sich um den Reiseantritt nach Berlin gemeinsam mit seinem Sohn Eugen um einer Einladung von Wilhelm von Humboldt zu folgen. Schon während der Verabschiedung von seiner Familie in Göttingen erfährt der Leser, dass das Verhalten des Professors nicht den kognitiven Fähigkeiten des größte[n] Mathematiker des Landes und somit auch nicht den Erwartungen des Lesepublikums von diesem entspricht, wodurch eine komische Wirkung dieser Darstellung entsteht:

Die Fahrt war qualvoll. Er nannte Eugen einen Versager, nahm ihm den Knotenstock ab und stieß mit aller Kraft nach seinem Fuß. Eine Weile sah er mit gerunzelten Brauen aus dem Fenster, dann fragte er, wann seine Tochter endlich heiraten werde. Warum wolle die denn keiner, wo sei das Problem?

Eugen strich sich die langen Haare zurück, knetete mit beiden Händen seine rote Mütze und wollte nicht antworten.

Raus mit der Sprache, sagte Gauß.

Um ehrlich zu sein, sagte Eugen, die Schwester sei nicht eben hübsch.

Gauß nickte, die Antwort kam ihm plausibel vor. Er verlangte ein Buch.

(Die Vermessung der Welt: 8f.)

Gauß` Laune ist gleich zu Beginn des Romans schlecht und bleibt es auch während der Reise. In dem Satz Die Fahrt war qualvoll werden keine Emotionen direkt beschrieben, aber er impliziert, dass der Protagonist sich körperlich und emotional ausgesprochen unwohl fühlt. Dies manifestiert sich auch in seinem weiterhin aggressiven Verhalten und den geringschätzigen Äußerungen über seine Kinder: Die abrupte Frage nach dem zivilen Stand seiner Tochter lässt annehmen, dass er wieder nur nach einem Grund sucht, um seinem Ärger freien Lauf zu lassen, der in Wirklichkeit eine ganz andere Ursache hat. Dass er von der Ehelosigkeit der jungen Frau nichts hält, drückt das Modalwort endlich aus, das laut Wahrig (Wahrig 2009:s.v. „endlich“) „,nach langer Erwartung, Verzögerung, nach langem Zweifel" bedeutet. Die Heirat ist also etwas, das seiner Meinung nach längst hätte stattfinden müssen. Die Begründung für das Ausbleiben eines geeigneten Kandidaten sieht Gauß in seiner Tochter selbst, denn er geht davon aus, dass sie von niemandem begehrt werde. Er äußert also seine Ungeduld darüber, dass sie noch nicht unter der Haube ist, was nur an einem ihm unbekannten Problem liegen könne. Er verwendet kein Personalpronomen, als er über sie spricht, sondern das Demonstrativpronomen die, was ebenfalls auf seine distanzierte Beziehung zu ihr schließen lässt. Überhaupt hat Gauß` Interrogativsatz eine doppelte Funktion. ${ }^{7}$ Er ist einerseits tatsächlich als Frage gemeint, in der sich der Sprecher nach den Gründen für die mangelnde Zahl an Heiratsanwärtern seiner Tochter erkundigt. Gleichzeitig macht er ihr damit den Vorwurf, keine Bewerber aufweisen zu können. Günthner $(2000,85)$ bezeichnet warum-Fragen als die ,,am häufigsten auftretenden syntaktischen Konstruktionen zur Vorwurfsverpackung“. Der Vorwurf wird hier abgeschwächt, weil Gauß immer behaupten kann, er habe sich nur nach den Gründen des Ausbleibens von Verehrern seiner Tochter erkundigt. Allerdings verstärkt die folgende Frage wo sei das Problem? die Missbilligung. Gauß manifestiert hiermit seine Ungeduld über die in seinen Augen nicht normale Situation, die nach den gesellschaftlichen Gepflogenheiten - und offensichtlich auch seiner Meinung nach - als negativ zu werden ist. Seine Empörung wird auch durch die Modalpartikel denn in der ersten Frage

Wolf (2015:54) verweist auf diese doppelte Funktion in einem Artikel über einen Dialog aus Franz Wedekinds Frühlings Erwachen. 
ausgedrückt. Helbig ( $\left.{ }^{3} 1994: 107\right)$ zufolge dient diese Partikel als „Indikator für den Sprechhandlungstyp Vorwurf: ,auf diese „Fragen“ werden nicht nur Antworten, sondern Rechtfertigungen erwartet.“

Eugen reagiert sichtlich erregt und zögert auch, diese „Frage“ zu beantworten. Dass er sich bedrängt fühlt, zeigt sich hier nicht durch eine Äußerung von ihm, sondern zum einen durch die Beschreibung der Körpersprache: er fährt sich durchs Haar und spielt nervös mit einem Gegenstand. Dies sind laut Kurzon Anzeichen für Verlegenheit (1998:36). Zudem verrät auch die Tatsache, dass er zunächst schweigt - ausgedrückt durch das Modalverb wollte -, sein Unbehagen (Vgl. Kurzon 1998:19 f.). Indem er nicht sofort antwortet, drückt er auch etwas aus: „In terms of a Peircian approach, silence as silence [...] is symbolic since it is a word that has arbitrarily conventional meaning" ". Eugens Stille ist kein Zufall, sondern auch eine Art Reaktion auf die Frage seines Vaters: „On being asked a question, the addressee has given a verbal stimulus, but $\mathrm{s} /$ he has a choice between speech and silence as the answer. This choice creates meaning, thus silence has meaning" (Kurzon 1998:25). Die bereits beschriebene Körpersprache ist ein Hinweis darauf, dass Eugen sich vor einer vorhersehbaren zornigen Reaktion seines Vaters fürchtet, durch die er wieder gedemütigt wird, und einen weiteren Konflikt vermeiden will. Sein Schweigen ist also zum einen die Darstellung einer hohen emotionalen Beteiligung und außerdem eine Strategie, größere Kontrolle über die Situation zu erlangen und sich nicht auf das „Spiel“ seines Vaters einzulassen (ibid., 35). Dieser hat kein Verständnis für die Verlegenheit seines Sohnes und fühlt sich offensichtlich davon provoziert. ${ }^{9}$ Kurzerhand befiehlt er ihm: „Raus mit der Sprache“ (8). Hier kommt zum ersten Mal direkte Rede vor, dies auch deshalb, weil diese performative Kurzformel nicht in gleicher Weise in indirekter Rede formuliert werden kann. Gauß`Aufforderung gewinnt so im Vergleich zur erlebten Rede an Schärfe. Die Äußerung ist zwar nicht durch ein Ausrufezeichen markiert, aber die elliptische Struktur trägt zum schroffen Ton des Gesagten bei. ${ }^{10}$ Mit dieser knapp formulierten Anordnung gibt Gauß seinem Sohn zu verstehen, dass er der mächtigere Gesprächspartner ist und daher eine Antwort von ihm einfordern kann. Er ist damit auch erfolgreich, aber Eugen erwidert dementsprechend vorsichtig. Anscheinend ist er auf eine negative Reaktion seines Vaters gefasst und setzt seiner Aussage daher die Wendung um ehrlich zu sein voran. Damit mildert er seine folgende Äußerung, um diese nicht zu grob klingen zu lassen. Die gleiche Funktion hat auch die Gradpartikel eben, welche die Verneinung abschwächt (vgl. Helbig ${ }^{3} 1994: 123$ ). Diese Strategie ist offensichtlich erfolgreich, denn Gauß stimmt ihm zu und geht nicht weiter auf das Thema ein. Stattdessen weist er ihn an, ihm ein Buch zu geben: Er verlangte ein Buch (8). Dies ist symptomatisch für die Vater-Sohn-Beziehung, in der der Vater Befehle erteilt und der Sohn zu gehorchen hat.

\section{Interpretation des Befundes}

Der Emotionsausdruck spielt in literarischen Texten eine große Rolle, um die Beziehungen der Figuren zueinander zu charakterisieren. Sowohl der Erzähler als auch die Figuren können Emotionen ausdrücken. Was den Erzähler betrifft, sind es vor allem der personale Erzähler und der ich-Erzähler, die mit ihren Emotionen das erzählte Geschehen kommentieren und glossieren (können). In dem Satz

Eine Weile sah er mit gerunzelten Brauen aus dem Fenster, dann fragte er, wann seine Tochter endlich heiraten werde

drückt der Erzähler aber keine Emotion aus, sondern beschreibt das emotionale Handeln einer Figur, nämlich Gauß. Dieser stellt eine Frage, die streng genommen keine Frage, sondern ein indirekter Sprechakt ist, denn er kennt die Antwort bereits selbst. Er möchte seine schlechte Laune zum Ausdruck bringen, die hier einen ganz anderen Ursprung hat, und der tut das mit einem indirekten

8 Kurzon (1998:23). Vgl. auch Kamper/Wulf (1992:7): „Schweigen ist in sozialen Situationen nicht bloß Leere; es vermittelt Bedeutungen.“

9 Vgl. Sifianou (1995:102); Kurzon (1998:26): „[...] if a person remains silent instead of expressing a positive feeling of, say, praise, this may be interpreted as dispraise.“

10 Ortner (1987:99 f.) spricht von „Ellipsen als Mittel der Affektsyntax“. 
Sprechakt, der seine Gefühle deutlich formuliert. Der Erzähler selbst drückt also keine Emotionalität aus, wohl aber will er durch die syntaktische Figur der indirekten Rede signalisieren, dass nicht er, sondern Gauß für den Inhalt der Rede verantwortlich sei. Eine direkte Rede würde diesen Gesichtspunkt nicht formulieren können. Überhaupt spielt die indirekte Rede bei Kehlmann eine große Rolle. Sein Roman lebt vor allem durch die Komik, die durch die Präsentation von illustren historischen Persönlichkeiten entsteht, die sich aber durchwegs wie unreife Kinder benehmen und somit lächerlich erscheinen. Die rhetorische Distanz des Erzählers trägt zu dieser Wirkung bei.

In der Erzählerrede werden hier kaum Emotionen unmittelbar dargestellt. Man kann deutlich sehen, dass direkte Äußerungen wie er war glücklich oder er war traurig selten sind. Es dienen eher andere sprachliche Mittel auf verschiedenen Ebenen, die Emotionalität ausdrücken oder beschreiben, so zum Beispiel Verben und verbale Phraseologismen, die körperliche (Re-)Aktionen schildern und benennen (Somatismen), wie die Augenbrauen zusammenziehen, sich verbeugen, die Achseln zucken, die Haare zurückstreichen und seine Mütze mit beiden Händen kneten. Dabei wird auf eine minutiöse Beschreibung von Details verzichtet, man kann zum Beispiel auf ganz verschiedene Weise und mit unterschiedlicher Wirkung die Augenbrauen zusammenziehen. Der Kontext trägt dazu bei, die gefühlsmäßige Befindlichkeit der Figuren deutlich zu machen. Das Gleiche gilt für das Berichten von Handlungen, die Rückschlüsse auf Emotionen liefern: Personen hören nicht $\mathrm{zu}$, schweigen, oder verlangen ein Buch.

Letzteres Beispiel gibt einen Hinweis auf die Art und Weise, wie Gauß seinen Sohn zur Übergabe eines Gegenstandes auffordert und somit auch auf eine gewisse Stimm-Modulation, wie sie bei Befehlen eingesetzt wird. Des Weiteren können lautliche Mittel in der schriftlichen Erzählersprache durch Verba dicendi ersetzt werden. In den oben zitierten Textausschnitten kommen ausschließlich das neutrale sagen und fragen zum Einsatz, aber als in der letzten beschriebenen Szene Gauß das erforderte Buch erhält und schließlich aus dem Fenster wirft, reagiert Eugen alles andere als erfreut: Das sei seines gewesen, rief Eugen (10). Hier werden mit dem Verb auch Stimm-Modulation und Lautstärke geschildert. Ein anderes Mittel ist die Verwendung von Adverbialen, um den lautlichen Aspekt der Sprechhandlung zu beschreiben. So werden hier beispielsweise Dinge müde oder säuerlich gesagt. In der Figurensprache hingegen gibt es verschiedene graphische Mittel wie Fettdruck, Unterstreichung etc. wobei in den hier herangezogenen Texten nur das Ausrufezeichen vorkommt.

Eine Charakterisierung eines stimmlichen Merkmales taucht auch in der Figurenrede auf, nämlich als Wilhelm Humboldt Gauß` knappen Kommentar zu seinem Beruf als enttäuscht geklungen beschreibt. Ansonsten findet sich der Emotionsausdruck in der Figurensprache in unmittelbarerer Form.

Eine Analyse der einschlägigen lexikalischen Mittel bestätigt den syntaktischen Befund (siehe unten): Der emotionale Ausdruck ist nur im Kontext interpretierbar. Adjektive wie traurig beziehen sich nicht unbedingt auf eine gefühlsmäßige Befindlichkeit, während Verben wie einfallen Hinweise auf die emotionale Einstellung des Sprechers liefern können. So können auch negativ konnotierte Wörter wie Pedanterie, notdürftig und ungerecht in anderen Situationen z.B. humorvoll und ironisch eingesetzt werden und dadurch dazu beitragen, positive Gefühle zu übermitteln. Das Gleiche gilt für Vergleiche und Metaphern, wie sie hier aus dem Bereich der Tierwelt vorliegen.

Wie die Beispiele aus den drei Textausschnitten gezeigt haben, tragen auch Wörter zum Emotionsausdruck bei, die überhaupt erst durch den Kontext eine Bedeutung erlangen, wie das vor Allem bei Partikeln der Fall ist. Modalpartikeln wie wirklich, endlich und denn verfügen zwar über eine gewisse Grundbedeutung, können aber völlig entgegengesetzte Gefühle zum Ausdruck bringen (Vgl. Wolf 2018:20). Das Gleiche gilt für Gradpartikeln wie gar und eben, denn um deren Sinn zu analysieren, muss überhaupt erst klar sein, was sie graduieren. Über Demonstrativpronomina heißt es, dass sie deiktische Funktion haben, aber in dem Beispiel Warum wolle die denn keiner wäre das Personalpronomen als Verweis auf die Tochter des Sprechers ausreichend. Durch die Verwendung des Demonstrativpronomens drückt er seine emotionale Einstellung zu ihr aus. Auch die oben erwähnte Interjektion ach so erhält in ihrem Kontext eine weitere Bedeutung als im Duden angegeben.

Die Tatsache, dass ein Sprecher negativen Gefühlen seines Gesprächspartners durch die Strategie der (De-)Emotionalisierung vorbeugen will, weist auf eine gewisse Beunruhigung hin. Um 
ungewünschte negative Emotionen zu vermeiden, gibt es ebenfalls zahlreiche sprachliche Mittel, so zum Beispiel formelhafte Wendungen im Vorvorfeld (Er wolle ja keinem zu nahe treten ..., um ehrlich zu sein...). Zur Beschwichtigung dienen hier auch die Fokuspartikel nur und die Modalpartikel ruhig sowie die Wiederholung der Responsivpartikel nein.

Durch den Kontext ist zwar feststellbar, ob Emotionen vorliegen und ob diese positiv oder negativ sind, aber sie lassen sich häufig nicht präzise bestimmen. Sprachliche Mittel auf verschiedenen Ebenen drücken in erster Linie Emotionalität der Sprecher aus und lassen erkennen, dass es sich um emotive Äußerungen handelt, ohne sich auf eine spezielle Emotion wie etwa Glück, Trauer, Liebe etc. zu konzentrieren. Lexikalische Mittel sind hier zumeist aufschlussreicher als syntaktische, aber auch wenn letztere generell unpräziser sind, verfügen sie dennoch potentiell über mehr Expressivität.

Ein hohes Potential an Emotionalität hat zum Beispiel alles, was sich im Vorvorfeld befindet. Es ist ein Unterschied ob jemand Der Typ nervt mich oder Der Typ, der nervt mich sagt, auch wenn der Inhalt der Proposition sich nicht verändert, denn die Extraposition nach links verfügt über einen wesentlich größeren emotionalen Gehalt. In dem Beispiel Um ehrlich zu sein, sagte Eugen, die Schwester sei nicht eben hübsch könnte der Sprecher den metakommunikativen Nebensatz durch eine Adverbiale ersetzten: Die Schwester sei ehrlich gesagt nicht eben hübsch. Dadurch käme allerdings sein bedenkliches Zögern nicht gleichermaßen zum Ausdruck.

Dass Eugen seine Befangenheit überwindet und auf die Frage seines Vaters antwortet, liegt an der Schärfe von dessen Aufforderung, sich zu äußern: Raus mit der Sprache. Selbst als Imperativsatz hätte der Appell nicht die gleiche Eindringlichkeit: Komm raus mit der Sprache, und als Deklarativsatz hätte er noch mehr an Expressivität verloren: Ich möchte, dass du mit der Sprache raus kommst. Hier zeigt sich, dass Deklarativsätze als syntaktisches Mittel über den geringsten Grad der emotionalen Erregung verfügen, während die verschiedenen Formen der syntaktischen Reduktion tendenziell eine wesentlich höhere Expressivität aufweisen, wenn es sich nicht, wie es das Beispiel Oder ein Deutscher den Humor zeigt, um Einsparung handelt, welche durch Vorerwähnung ermöglicht wird. Auch hier wird nur durch den Kontext erkenntlich, ob die Sprachökonomie der Steigerung der Expressivität dient oder zu einem anderen kommunikativen Zweck.

Ohne einen solchen Zusammenhang lässt sich auch nicht entscheiden, ob etwa ein Interrogativsatz (nur) dazu dient, eine Frage zu formulieren, sondern auch um eine emotionale Einstellung zum Ausdruck zu bringen. Vor allem, wenn eine Modalpartikel, wie in dem Beispiel Warum wolle die denn keiner? vorkommt, liegt mit großer Wahrscheinlichkeit eine expressive Äußerung vor.

\section{4. $\quad$ Fazit}

Das letzte Beispiel zeigt wiederum, dass das Zusammenspiel aller sprachlichen Mittel, nämlich der lautlichen bzw. graphischen, lexikalischen und syntaktischen, beim Ausdruck von Emotionen eine Rolle spielt. Dabei wird deutlich, dass sich durch lexikalische Mittel Emotionen präziser bestimmen lassen, syntaktische Mittel aber potentiell über mehr Expressivität verfügen. Gerade deshalb ist es sinnvoll, mit einem kleinen Korpus zu arbeiten, um diese durch den Kontext zu erfassen. Sprachwissenschaft, wenn man sie ernst nimmt, unterliegt nicht einer außersprachlichen oder sprachfernen Logik, sondern muss die sprachinterne Logik aus der Sprachverwendung rekonstruieren. Menschliche Phänomene wie Modalität oder nur Emotionalität sind nicht durch eindeutige und unabänderliche sprachliche Zeichen encodiert, sondern müssen immer aus dem Text heraus analysiert und beschrieben werden.

\section{Literaturverzeichnis}

\section{Primärliteratur:}

Kehlmann, Daniel (22010): Die Vermessung der Welt. Hamburg.

\section{Sekundärliteratur:}

BALly, Charles ( $\left.{ }^{4} 1965\right)$ : Linguistique générale et linguistique française. Bern. 
Bestgen, Yves (1994): "Can emotional valence in stories be determined from words". In: Cognition \& Emotion, Nr. 8.1, S. 21-36. Zugänglich unter: http://www.tandfonline.com/doi/ abs/10.1080/02699939408408926 [30.10.2017]

BRunot, Ferdinand (1922): La pensée et la langue. Paris.

BüHLER, Karl (1934): Sprachtheorie, Die Darstellungsfunktion der Sprache. Jena.

Coseriu, Eugenio ( $\left.{ }^{3} 1994\right)$ : Textlinguistik. Eine Einführung. Tübingen; Basel.

DARwin, Charles ( $\left.{ }^{3} 1877\right):$ Der Ausdruck der Gemüthsbewegungen bei dem Menschen und den Thieren. Übers. von J. Victor Carus. Stuttgart. Zugänglich unter: https://de.wikisource.org/wiki/ Der Ausdruck der Gem\%C3\%BCthsbewegungen bei dem Menschen und den Thieren [17.01.2021]

Ekman, Paul / Friesen, Wallace V. (2003): Unmasking the face. A guide to recognizing emotions from facial clues. Cambridge, Massachusetts.

GüNTHNER, Susanne (2000): Vorwurfsaktivitäten in der Alltagsinteraktion. Grammatische, prosodische, rhetorisch-stilistische und interaktive Verfahren bei der Konstitution kommunikativer Muster und Gattungen. Tübingen.

HeLbig, Gerhard ( $\left.{ }^{3} 1994\right)$ : Lexikon deutscher Partikeln. Leipzig.

Herding, Klaus / Stumphaus, Bernhard (2004): Pathos, Affekt, Gefühl. Die Emotionen in den Künsten. Berlin.

JAKOBSOn, Roman (2007): Poesie der Grammatik und Grammatik der Poesie. Band 1. Poetologische Schriften und Analysen zur Lyrik vom Mittelalter bis zur Aufklärung. Hrsg. Sebastian Donat; Hendrik Birus. Berlin.

KAMPER, Dietmar / Wulf, Christoph, Schweigen (1992): Unterbrechung und Grenze der menschlichen Wirklichkeit. Berlin.

Konstantinidou, Magdalane (1997): Sprache und Gefühl: semiotische und andere Aspekte einer Relation. Hamburg.

Kurzon, Dennis (1998): Discourse of Silence. Amsterdam, Philadelphia.

LAKOFF, George / Mark Johnson (2003): Metaphors We Live by. Chicago.

Métrich, René / Faucher, Eugène (2009): Wörterbuch deutscher Partikeln. Unter Berücksichtigung ihrer französischen Äquivalente. Berlin.

Morris, Desmond (1994): Bodytalk. A World Guide to Gestures. Oxford.

OAtLey, Keith (2004): Emotions: A Brief History. Oxford [u.a.], Blackwell.

Ortner, Hanspeter (1987): Die Ellipse: Ein Problem der Sprachtheorie und der Grammatikbeschreibung. Tübingen.

SCHWARZ-FrIESEL, Monika (22013): Sprache und Emotion. Tübingen.

Sifianou, Maria (1995): "Do we need to be silent to be extremely polite? Silence and FTAs". In: International Journal of Applied Linguistics, Nr. 5.1, S. 95-110. Zugänglich unter: http://onlinelibrary.wiley.com.ezproxy.normandie-univ.fr/doi/10.1111/j.14734192.1995.tb00074.x/epdf [09.11.2017]

WAHRIG ( $\left.{ }^{9} 2012\right):$ Deutsches Wörterbuch. Gütersloh; München.

WiLCE, James M. (2009): Language and Emotion. Cambridge.

Wolf, Norbert Richard (2015): „,Sie, ich hätte es nicht vermocht, dir ein Wort zu sagen. Monologische Dialoge und Dialogstrukturen in Wedekinds ,Frühlings Erwachen““. In: Acta Facultatis Philosophicae Universitatis Ostraviensis. Studia Germanistica. Nr. 16, Ostrava, S. 53-60. Zugänglich unter: http://periodika.osu.cz/studiagermanistica/dok/archiv/2015_Studia-Germanistica-16.pdf [17.01.2021]

Wolf, Monika (2018): „,Na ja, Ideen muß man haben. 'Emotionsausdrückende Partikeln im Deutschen und ihre Entsprechungen im Französischen am Beispiel der Partikel ja“. In: Acta Facultatis Philosophicae Universitatis Ostraviensis. Studia Germanistica. Nr. 23, Ostrava, S. 53-60. Zugänglich unter: https://dokumenty.osu.cz/ff/studiagermanistica/archiv/2018_Studia-Germanistica-23.pdf [17.01.2021]

URL: https://www.duden.de [16.12.2020] 